\title{
EDITORIAL
}

\section{Editorial zum Schwerpunktthema: Lehrkräftemangel und Lehrerrekrutierung - historische Perspektive und aktuelle Problemlage}

\section{Editorial to the Focus Topic: \\ Teacher Shortage and Teacher Recruitment - Historical Perspective and Current Range of Problems}

Wie unter einem Brennglas, so die vielfach genutzte Metapher, habe die pandemieartige Ausbreitung des Virus SARS-CoV2/Covid 19 die Probleme in den verschiedenen gesellschaftlichen Bereichen gezeigt. Dies gilt auch für den Bildungsbereich. Im Zuge des Lockdowns und der damit verbundenen Einschränkungen einschließlich der Umstellung des Unterrichts auf den Einsatz digitaler Medien und Kommunikationsformen sind die infrastrukturellen, sachlichen und personellen Probleme in den Schulen überdeutlich geworden.

Allerdings richtet(e) sich die Kritik im Zuge der öffentlichen Diskussion hauptsächlich gegen die Lehrkräfte und ihre unzureichenden, wenn nicht sogar fehlenden digitalen Kompetenzen und auf die mangelhafte digitale Ausstattung der Schulen, nicht aber auf den schon lange zuvor diskutierten offensichtlichen Lehrermangel und die bisher als politische „Lösung“ angesehenen Sondermaßnahmen in der Lehrerausbildung (vgl. u. a. Dedering, 2020; Puderbach, Stein \& Gehrmann, 2016; Tillmann, 2019). Darauf, dass eine „Lösung“ andere, grundsätzlicher ansetzende politische Entscheidungen erfordern würde, verweist die derzeit in der Presse diskutierte Studie „Lehrkräftebildung - Wege aus der föderalen Sackgasse“ von Mark Rackles (2020), der als Staatssekretär acht Jahre in der Berliner Senatsverwaltung für Bildung, Jugend und Familie tätig war. Er sieht „den eigentlichen Schaden [...] darin, dass die Länder im Bildungsbereich die länderübergreifende Perspektive verloren haben und es keine eingeübte Praxis der verbindlichen Kooperation gibt“" (siehe auch GEW, 24.09.2020).

Diese Sondermaßnahmen und die - offensichtlich nicht erfolgte - Koordination der Maßnahmen zwischen den Bundesländern stehen im Mittelpunkt des vorliegenden Heftes. Um die Unterrichtsversorgung sicherzustellen, hat die Bildungspolitik zunehmend mehr Personen, die kein reguläres Lehramtsstudium absolviert, aber im Rahmen ihres Studiums schulrelevante Fachkenntnisse erworben haben, den 
Zugang zum Lehrerberuf eröffnet. Der Anteil dieser je nach Qualifikationsform als Quereinsteigende oder Seiteneinsteigende bezeichneten Personen an allen Lehrkräften variiert erheblich zwischen den Bundesländern - besonders hoch ist er derzeit mit jeweils mehr als 40 Prozent in Berlin und Sachsen, besonders niedrig z. B. in RheinlandPfalz (vgl. KMK, 2018).

Folgt man der derzeitigen Diskussion zu den bisher vorliegenden wissenschaftlichen Studien, so entsteht der Eindruck, als handele es sich um ein plötzlich aufgetretenes, aktuelles Problem. Ein Blick in die Geschichte des Lehrer*innenberufs in Preußen, wie ihn Bernd Zymek und Ulrich Heinemann mit ihrem Beitrag bieten, zeigt hingegen, dass die in diesem Heft dargestellten Probleme altbekannt sind und die jeweils getroffenen Sonder- und Notmaßnahmen nur bedingt wirksam waren. Der von den Autoren gewählte Ausschnitt aus der Geschichte der Maßnahmen zur Regulierung des „Lehrkräftearbeitsmarktes“ reicht vom 19. bis weit in die zweite Hälfte des 20. Jahrhunderts. Je nach historischem Zeitpunkt und der jeweiligen politisch-gesellschaftlichen Situation wurden die Maßnahmen angepasst, aber zu keinem Zeitpunkt sind Konzepte entwickelt worden, wie solche Krisen in Zeiten des Friedens, wenn nicht verhindert, so doch abgemildert werden könnten. Die Autoren setzen zwei Schwerpunkte: Zum einen zeigen sie, dass und warum es immer wieder Phasen von Lehrermangel und Lehrerüberschuss gab und wie sich dies professionspolitisch ausgewirkt hat. Zum anderen gehen sie speziell auf die von diesen Krisensituationen besonders geprägte Professionsgeschichte der Lehrerinnen ein. Der Blick in die Geschichte hält keine Lösung des aktuellen Problems bereit, aber er macht deutlich, dass es nicht nur darum gehen kann, Problemlagen durch Sondermaßnahmen abzumildern, sondern dass es neuer Konzepte bedarf, die in Kenntnis dessen, dass es auch zukünftig zu Perioden von Lehrermangel oder Lehrerüberangebot kommen kann, vorausschauend neue reguläre Wege der Zusatz- oder Nachqualifizierung für einen Einstieg in den Lehrer*innenberuf eröffnen.

In den beiden anderen Artikeln zum Themenschwerpunkt „Lehrkräftemangel und Lehrerrekrutierung" geht es um die aktuelle Situation. Torben Güldener, Nils Schümann, Ivonne Driesner und Mona Arndt haben in ihrem Projekt „Studienerfolg und -misserfolg im Lehramtsstudium" untersucht, wie viele derjenigen, die sich zunächst für ein Lehramtsstudium entschieden haben, im Laufe desselben „verloren“ gehen und wie sich dieser Schwund erklären lässt. Sie haben seit 2017 reale Studienverläufe von Lehramtsstudierenden in Mecklenburg-Vorpommern analysiert und zusammen mit Daten der amtlichen Statistik und bisherigen Forschungserkenntnissen analysiert. Insgesamt zeigt sich, dass sich die Abbruch- und Erfolgsquoten der Universitäten in Mecklenburg-Vorpommern nicht mit den bundesweiten Quoten decken. Die Schwundquoten dieses Bundeslandes sind deutlich höher als in den anderen Bundesländern. Vor allem im Sekundarbereich scheidet ein Großteil des Lehrkräftenachwuchses aus den Kohorten aus. Viele dieser Studierenden bleiben allerdings an den Hochschulen, aber sie wechseln den Studiengang oder die Studienfächer. 
Im Mittelpunkt des dritten Beitrags zum Themenschwerpunkt steht die Gruppe der Seiteneinsteiger*innen. Gabriele Bellenberg, Christoph Bressler, Christian Reintjes und Carolin Rotter haben sie als eine besondere Gruppe, d.h. in Abgrenzung von jener der Quereinsteigenden, untersucht. Ihr Interesse gilt der Frage, wie die Schulleitungen und die Ausbildungsbeauftragten, die für die schulische Ausbildung sowohl der Referendar*innen und der Quereinsteiger*innen als auch der Seiteneinsteiger*innen verantwortlich sind, letztere anleiten und beraten. Auf der Grundlage von Interviews an vier Schulen in Nordrhein-Westfalen mit mehreren Bildungsgängen präsentieren sie erste Ergebnisse zu der Frage, wie Schulen die Rekrutierung und die Qualifizierung von Seiteneinsteiger*innen gestalten. Nach ihren Ergebnissen erweist sich der Seiteneinstieg als ein positiv zu bewertendes Instrument, um dem Lehrermangel entgegenzuwirken. Die Autor ${ }^{\star}$ innen richten die Aufmerksamkeit vor allem auf die - zum Teil neuen - innerschulischen Ausbildungs- und Kooperationsstrukturen, die mit dem Ziel etabliert wurden, die im Vergleich zum grundständigen Referendariat knappe Ausbildungszeit der Seiteneinsteigenden auszugleichen. Die Befunde werden abschließend aus einer professionstheoretischen Perspektive diskutiert.

Mit dem Bericht von Ivonne Driesner und Mona Arndt wird der Versuch einer Systematisierung der derzeitigen Qualifizierungsmaßnahmen für Lehrkräfte ohne Lehrbefähigung unternommen. Auf Basis von öffentlich zugänglichen Informationen der einzelnen Bundesländer wird zunächst eine Typologie der Qualifizierungen nicht grundständig ausgebildeter Lehrkräfte entwickelt, so dass die Unterschiede zwischen Seiten- und Quereinstieg hinsichtlich des Ausbildungsangebots und des Status der entsprechend Ausgebildeten deutlich werden. Im Ergebnis zeigt sich, dass vor allem der Seiteneinstieg sehr unterschiedlich gestaltet wird, u. a. mit der Folge, dass in etlichen Bundesländern eine Gleichstellung mit grundständig ausgebildeten Lehrkräften nicht möglich bzw. nicht vorgesehen ist. Die Autor*innen weisen zugleich darauf hin, dass die Programmanalyse nicht erlaubt, gesicherte Aussagen über die Qualität der Lehrkräftebildung qua Seiteneinstieg - und damit über die Qualität des Unterrichts zu machen. Hierzu seien weitere Forschungen nötig.

Harald Freiling beschäftigt sich in seinem Bericht mit den „Vertretungslehrkräften“, einer heterogenen Gruppe von Lehrkräften, die generell, aber auch in der gegenwärtigen Diskussion über Lehrkräftemangel kaum beachtet wird. Bei den Vertretungslehrkräften handelt es sich um Personen, die zwar nicht als Lehrer ${ }^{\star}$ innen ausgebildet worden sind, aber dennoch eine auch pädagogisch relevante Ausbildung absolviert haben, angefangen von Erzieher ${ }^{\star}$ innen bis zu Musikwissenschaftler ${ }^{\star}$ innen. Sie werden in vielen Schulen mit zeitlich immer neu befristeten Verträgen und einem eher dürftigen Gehalt beschäftigt. Im Unterschied zu den Seiten- oder Quereinsteigenden wird ihnen keine Qualifizierung und damit auch keine berufliche Perspektive geboten. Es geht keineswegs um eine kleine Minderheit, die in unseren Schulen unter solchen Verhältnissen arbeitet. Die Beschreibung ihrer prekären Situation verbindet der Autor 
mit Forderungen, auch diesen Lehrkräften eine berufsbegleitende Qualifizierung anzubieten und ihnen so einen Einstieg in eine geregelte Berufslaufbahn zu ermöglichen.

Klaus-Jürgen Tillmann fragt in seinem Beitrag zur „Diskussion“, in welchem Ausmaß die Handlungen der bildungspolitisch Verantwortlichen mitverursachend für die gegenwärtige Krise sind. Im ersten Teil geht er auf die Möglichkeiten und Grenzen staatlicher Lehrerbedarfsprognosen ein und kommt zu dem Schluss, dass der aktuelle Lehrermangel weniger auf Fehler der Administration bei der Planung als vor allem auf die nicht vorhersehbare Steigerung von Geburten- und Zuwandererzahlen und auf die ungünstigen quantitativen Entwicklungen beim studierenden Nachwuchs zurückzuführen sei. Hier zeigten sich erhebliche Mängel bei den Prognosen, z. B. im zeitlichen Ablauf, die dringend behoben werden müssten. Im zweiten Teil befasst der Autor sich mit der Sondermaßnahme des „Seiteneinstiegs“, die in vielen Bundesländern den Lehrkräftemangel zwar entschärft habe, mit der aber in Kauf genommen werde, dass eine große Zahl von nur mäßig qualifizierten und weitgehend unerfahrenen Kräften nun über lange Zeit mit hoher Stundenzahl unterrichtet. Als Alternative zu den verschiedenen Sondermaßnahmen auf Zeit entwirft der Autor einen als dauerhaft zu etablierenden Studiengang für den Seiteneinstieg, in dem auch die notwendigen pädagogischen und didaktischen Qualifikationen zu vermitteln wären.

Dass für die Qualität von Bildung und Erziehung nicht nur gut qualifizierte und in ausreichendem Maße vorhandene Lehrkräfte entscheidend sind, sondern auch die bedarfsgerechten Ressourcenzuweisungen an die Schulen, ist das Thema des Beitrags von Caroline Gröschner. Sie belegt dies anhand ihrer Untersuchung zum Umgang von Grundschulleitungen mit diesem Instrument der bildungspolitischen Steuerung und deren Einstellungen dazu.

Auch in dem Bericht von Sebastian Stark und Barbara Lochner geht es um die Bedeutung eines sozial- und bedarfsorientierten Einsatzes der finanziellen Mittel. Sie fokussieren hierbei auf den vorschulischen Bereich und die Mittel, die den deutschen Bundesländern im Rahmen des sogenannten „Gute-KiTa-Gesetzes“ für die Qualitätsverbesserung in der Kindertagesbetreuung vom Bund zusätzlich bereitgestellt worden sind.

Götz Bieber, Kathrin Dedering, Marianne Krüger-Potratz \& Klaus-Jürgen Tillmann

\section{Literatur und Internetquellen}

Dedering, K. (2020). Quer-/Seiteneinsteigende in den Lehrerberuf im Spiegel der empirischen Forschung. Themenbereiche, Befunde und Desiderata. DDS - Die Deutsche Schule, 112 (1), 91-104. https://doi.org/10.31244/dds.2020.01.06 
GEW (Gewerkschaft Erziehung und Wissenschaft). (2020, 24.09.). Staatsvertrag zur Deckung des Lehrkräftebedarfs. Zugriff am 13.10.2020. Verfügbar unter: https://www.gew.de/aktuelles/detailseite/neuigkeiten/staatsvertrag-zur-deckung-des-lehrkraeftebedarfs.

KMK (Sekretariat der Ständigen Konferenz der Kultusminister der Länder in der Bundesrepublik Deutschland). (2018). Einstellung von Lehrkräften 2017. Tabellenauszug. Berlin: KMK.

Puderbach, R., Stein, K., \& Gehrmann, A. (2016). Nicht-grundständige Wege in den Lehrerberuf in Deutschland - eine systematisierende Bestandsaufnahme. Lehrerbildung auf dem Prüfstand, 9 (1), 5-30.

Rackles, M. (2020, 08.10.). National, föderal ... ganz egal? Die Lehrkräftebildung ist ein Lackmustest für den Bildungsföderalismus. Zugriff am 12.10.2020. Verfügbar unter: https://www.jmwiarda.de/2020/10/08/national-föderal-ganz-egal/.

Tillmann, K.-J. (2019). Von einer Notmaßnahme zu einem dauerhaften Konzept? Der Seiteneinstieg in den Lehrerberuf. Pädagogik, 71 (6), 11-14.

https://doi.org/10.31244/dds.2020.04.01 